\title{
CARDIOVASCULAR THERAPEUTIC PROPERTIES OF NANOSTRUCTURED HYDROGELS
}

\author{
Astrid Varela Arzate ${ }^{*}$, Aline Ruiz Ceja ${ }^{2}$, Laura Cornejo Cornejo ${ }^{3}$ \\ ${ }^{1}$ CICS-UMA IPN. E-mail: astrid_120@live.com.mx \\ ${ }^{2}$ ESM-IPN. \\ ${ }^{3}$ ESM- IPN.
}

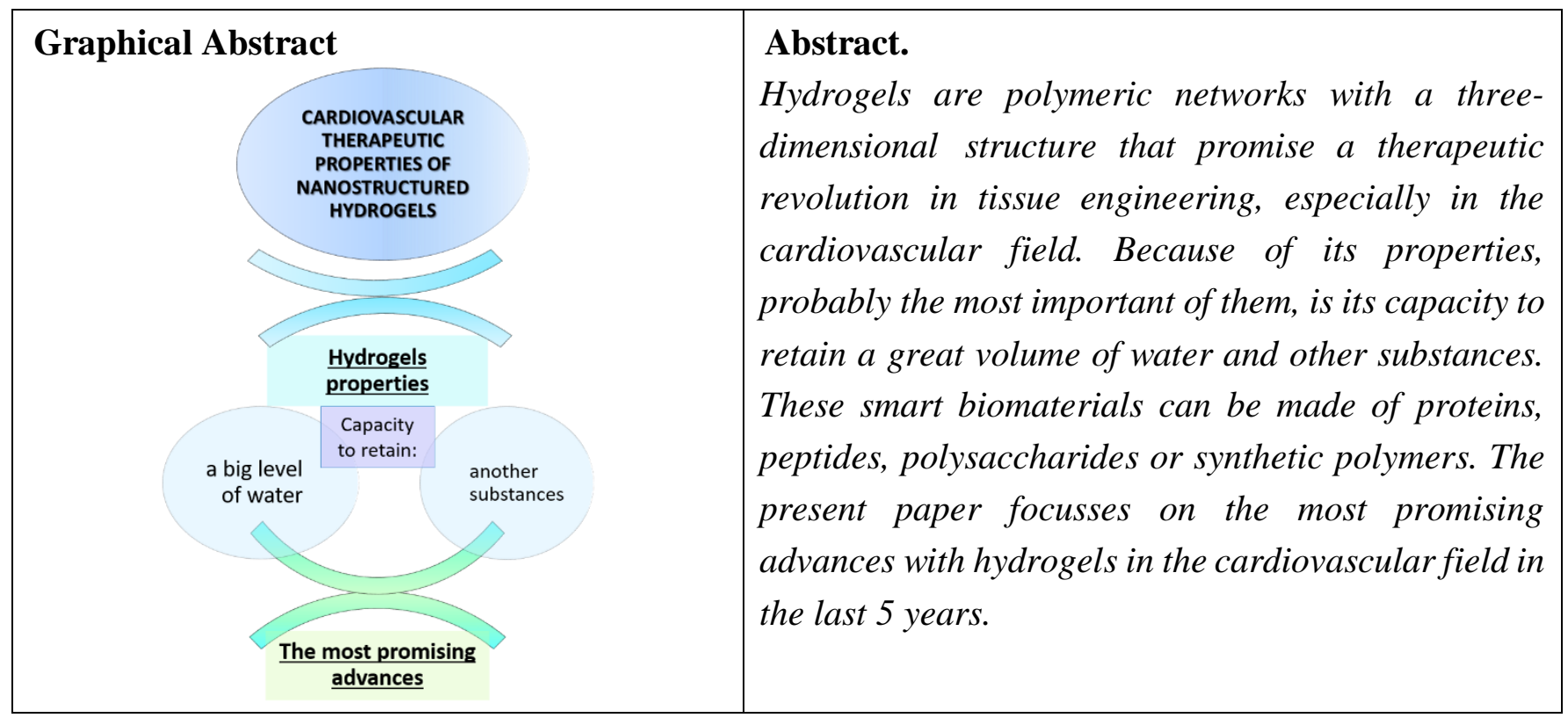

Keywords: hydrogel, tissue, nanostructured, cardiovascular.

\section{INTRODUCTION}

Hydrogels are polymeric networks with a three-dimensional structure that promise a therapeutic revolution in tissue engineering, especially in the cardiovascular field; due to its properties, probably the most important of them, its capacity to retain a great volume of water and another substances, they are key to advance in tissue engineering and drug delivery, on demand with hydrogels with the capacity of repeat answer to light, thermal and electric stimuli [1,2]. One example, is the study published in 2018 by Tal Dvir and co-workers that exposed autologous tissue implants by removing tissue pieces from patients, the cells were reprogrammed to become pluripotent stem cells, the extracellular matrix was processed into an immunologically compatible thermosensitive hydrogel. This initiated the generation of functional implants that help regenerate tissue with a minimal risk of immunological rejection. [3] These smart biomaterials can be made of proteins, peptides, polysaccharides or synthetic polymers. 
In a particular way, adding carbon nanostructures to hydrogels has a promising future by virtue of their multiform and electronic capacity. [1]

\section{CARDIOVASCULAR THERAPEUTIC PROPERTIES}

In this section we expose some of the most innovative advances with hydrogels in the cardiovascular field in the last 5 years.

\subsection{Carbohydrate hydrogels:}

Polysaccharides are extremely advantageous being widely present in living organisms and often being produced by recombinant DNA techniques. In fact, they even may offer an economical advantage over synthetic polymers considering coming from renewable sources. [1]

2.1.1 The birth to a new generation of implants: Polysaccharides like chitosan, alginate, hyaluronic acid, cellulose and agarose are used to form gel biomaterials. It was demonstrated that the addition of carbon nanofibers into porous chitosan scaffolds to develop a highly conductive compound that was seeded with neonatal rat heart cells and cultivated up to 14 days without electrical stimulation, enhanced the properties of cardiac tissue constructs. Apparently due to an enhanced transmission of electrical signals between the cells. In addition, the incorporation of carbon nanofibers also resulted in the highest expression of specific genes from heart tissue that are involved in muscle contraction and electrical coupling. This can reconstruct natural tissue that has been exposed to high mechanical stress or that requires electrical and thermal conductivity at physiological state. [4]

2.1.2 Innovation of myocardial patches: The main limitation of current myocardial patches that hinder the transfer of electrical signals between myocardiocytes is the risk of inducing arrhythmias. One way to repair cardiovascular defects with a minimal of this, is the addition of subtoxic concentrations of carbon nanotubes in a single wall incorporated in a gelatin-chitosan hydrogel, acting as nanobridges, which would result in an improvement of the electrical conductivity with a consequent synchronous beat. [5]

\subsection{Synthetic polymer hydrogels}

2.2.1 Disclosure of injectable delivery system for vasculogenesis and cardiac repair: The development of a biocompatible hydrogel with two compounds: a proangiogenic gene of graphene oxide nanometer (GO) and vascular endothelial growth factor-165 (VEGF) for myocardial therapy, was injected into rats with myocardial infarction, intramyocardial route, in peri-infarct regions. The VEFGF secreted from transfected cardiomyocytes in vitro, demonstrated profound mitotic activities in the endothelial cells with a significant increase in the density of the myocardium in the injected peri-infarct region and a reduction in the area of the scar in the hearts, suggesting the usefulness of a combined system of hydrogel-based gene therapy for ischemic heart diseases. [6]

\subsection{Polypeptide hydrogels}

Polypeptide is an ideal choice for biomaterials due to their biodegradability and biocompatibility. [1] 2.3.1 Modernization of cardiac repair using peptide hydrogels from human umbilical cord mesenchymal stem cell derived exosomes: In 2019 Chaoshan Han and co-workers showed the result of a study that demonstrated peptide PA-GHRPS protects $\mathrm{H} 9 \mathrm{c} 2$ cells from oxidative stress induced by $\mathrm{H}_{2} \mathrm{O}_{2}$. This peptide is gelatinized better with another one called NapFF. These two peptides formed the hydrogel that was used for encapsular exosomes, which were recognized as a potential therapy for cardiovascular disease. These help to 
maintain them stable and sustained release. This mixture was injected into the area of the infarcted edge of the hearts of the rat, and were compared to the ones that received treatment with exosomes without hydrogel. The hydrogel mixture improved myocardial function by reducing inflammation, fibrosis and apoptosis, and by promoting angiogenesis. [7]

2.3.2 Novel result with the injection of a recombinant human collagen hydrogel to improve cardiac function and reduce pathological remodeling after an acute myocardial infarction: According to the results of a study published in 2018 by Sarah McLaughlin and co-workers, injectable hydrogels of recombinant human collagen type I (rHC) and type III (rHC III) restored heart function in mice and simultaneously repaired infarction, because pathological remodeling of the cardiac extracellular matrix after myocardial infarction is a key factor contributing to heart failure. [8]

\section{CONCLUSION}

The use of hydrogels nanostructures in cardiovascular field is still young, however, this topic has fascinated day by day medical chemists all around the world. Hydrogels suggest promising alternatives to solve several dilemmas that actual therapeutics have shown, some of them even with better results.

\section{REFERENCES}

[1] Iglesias, D.; Bosi, S., Melchionna, M.; Marchesan T. and S. The glitter of carbon nanostructures in hybrid/composite hydrogels for medicinal use. Current Topics in Medical Chemestry, 2016, 16, 18761989

[2] Croisfelt, F.M.; Tundisi, L.L.; Ataide, J.A.; Silveira, E.; Tambourgi, E.B.; Jozala, A.F.; Souto, E.M.; Mazzola, P.G. Modified-release topical hydrogels: a ten-year review. Journal of Materials Science. 2019, 54 (16), 10963-10983.

[3] Edri, R.; Gal, I.; Noor, N.; Harel, T.; Fleischer, S.; Adadi, N.; Green, O.; Shabat, D.; Heller, L.; Shapira, A.; Gat-Viks, I.; Peer, D.; Dvir, T. Personalized Hydrogels for Engineering Diverse Fully Autologous Tissue Implants. Advanced materials, 2018, 31 (1) 1803895.

[4] Martins, A. M.; Eng, G.; Caridade, S. G.; Mano, J. F.; Reis, R. L.; Vunjak-Novakovic, G. Electrically conductive chitosan/carbon scaffolds for cardiac tissue engineering. Biomacromolecules, 2014, 15 (2), 635-643

[5] Pok, S.; Vitale, F.; Eichmann, S. L.; Benavides, O. M.; Pasquali, M.; Jacot, J. G. Biocompatible carbon nanotube-chitosan scaffold matching the electrical conductivity of the heart. ACS Nano, 2014, 8 (10), 9822-9832.

[6] Paul, A.; Hasan, A.; Kindi, H. A.; Gahardwar, A. K.; Rao. V. T. S.; Nikkhah, M.; Shinin, S. R.; Krafft, D.; Dokmeci, M. R.; Shum-Tim, D.; Khademhosseini, A. Injectable graphene oxide/hydrogelbased angiogenic gene delivery system for vasculogenesis and cardiac repair. ACS Nano, 2014, 8 (8), 8050-8062.

[7] Han, C.; Zhou, J.; Liang, C.; Liu, B.; Pan, X.; Zhang, Y.; Wang, Y.; Yan, B.; Xie, W.; Liu, F.; Yu X.Y.; Li, Y. Human umbilical cord mesenchymal stem cell derived exosomes encapsulated in functional peptide hydrogels promote cardiac repair. Biomaterials Science, 2019, 7 (7) 2920-2933.

[8] McLaughlin, S.; McNeill, B.; Podrebarac, J.; Hosoyama, K.; Seymour, R.; Liang, W.; Ruel, M.; Suuronen, E.; Alarcon, E. Injection of a recombinant human collagen hydrogel improves cardiac function and reduces pathological remodeling post myocardial infarction. Journal of Molecular and Cellular Cardiology. 2018, 124, 104. 